\title{
Comprehensive parametrization of surface-layer transfer coefficients for use in atmospheric numerical models
}

\author{
Hendrik Wouters • Koen De Ridder • \\ 4 Nicole P. M. van Lipzig
}

Abstract A new non-iterative bulk parametrization for surface-layer transfer coefficients for momentum and heat is presented. It is applicable for a wide range of aerodynamic and thermal roughness lengths, and includes the effect of 10 the roughness sublayer. As a consequence, the non-iterative method is suitable ${ }_{11}$ for every surface type, especially for urban surfaces for which existing non12 iterative parametrizations fail. The analytical approximation compares very 13 well with an iterative approach. Our method can be easily implemented in 14 atmospheric numerical models that already employ a non-iterative approach.

Keywords Monin-Obukhov Similarity Theory - Surface energy balance • Surface-layer transfer coefficients - Thermal roughness length - Turbulent fluxes

\section{$18 \quad 1$ Introduction}

19 Turbulent fluxes of momentum, sensible heat and latent heat from the surface 20 to the atmosphere largely determine the interaction between the surface and 21 the air above. Consequently, they have a substantial affect on wind, tempera22 ture, moisture and concentrations of other air constituents near the surface. A 23 common approach to calculating the surface transfer coefficients and the conse24 quential fluxes is by means of the Monin-Obukhov similarity theory (MOST).

25 Within this framework, transfer coefficients generally need to be calculated 26 iteratively. As iterative solutions for the transfer coefficients are computation-

H. Wouters · K. De Ridder

VITO-Flemish Institute for Technological Research, Boeretang 200, 2400 Mol, Belgium E-mail: hendrik.wouters@vito.be

H. Wouters · N. P. M. van Lipzig

KU Leuven, dept. Earth and Environmental Sciences, Celestijnenlaan 200E, 3001 Leuven, Belgium. E-mail: hendrik.wouters@ees.kuleuven.be 
ally expensive, analytical approximations have been investigated for the past 30 years.

To simplify this problem, early analytical approximations such as Louis (1979), Byun (1990), Lee (1997) and Viterbo et al. (1999) assume $z_{0 \mathrm{H}}=z_{0}$ ( $z_{0}$ and $z_{0 \mathrm{H}}$ are the aerodynamic and thermal roughness lengths, respectively). Others assume a constant $z_{0 \mathrm{H}} / z_{0}$ ratio, or a fixed $k B^{-1}=\ln \left(z_{0} / z_{0 \mathrm{H}}\right)$, as in Pleim (2006). More sophisticated methods such as Garratt (1992, see p. 243), Uno et al. (1995), Launiainen (1995), Holtslag and Ek (1996), Mascart et al. (1995), Song (1998), De Bruin et al. (2000), Blümel (2000) and Li et al. (2010) allow a varying $k B^{-1}$. A few of the above-mentioned analytical approaches have been compared in van den Hurk and Holtslag (1997). As stated by their respective authors, the analytical approaches above can reproduce the iterative procedure very well yet for limited ranges of $z / z_{0}(z$ is the reference height, or typically the height of the first vertical model level), $k B^{-1}$ and/or $R i_{\mathrm{B}}$ (bulk Richardson number). However, they fail to reproduce the full range $-0.5 \leq k B^{-1} \leq 30,10 \leq z / z_{0} \leq 10^{5}$ and $-5.0 \leq R i_{\mathrm{B}} \leq 2.5$. In particular, the case of urban surfaces, for which very high $k B^{-1}$ values of more than 10 (see Voogt and Grimmond, 2000; Sugawara and Narita, 2008) and very low $z / z_{0}$ values down to 10 are found, is problematic. As the horizontal resolution of atmospheric models increases, urban areas may well extend over several grid boxes. Therefore, a new analytical parametrization for turbulent fluxes applicable for this situation is necessary.

We present herein new bulk transfer coefficients for both unstable and stable surface-layer conditions as an approximation to the iterative procedure within the MOST framework. As opposed to existing parametrizations, they are applicable for $10 \leq z / z_{0} \leq 10^{5}$ and $-0.5 \leq k B^{-1} \leq 30$ that capture most, if not all, surface types and model configurations. They also account for the roughness-sublayer (RSL) effect, which is important when $z / z_{0}$ becomes small (see Garratt, 1992, section 3.3.4). The advantage of an analytical form is easy implemention in models that already use an analytical approach. In addition, analytical formulations avoid slow or non-convergence that can occur with iterative procedures.

The structure of the paper is as follows: a summary of the MOST is given in Section 2 including a brief description of the iterative procedure. Section 3 describes the new analytical approximation, and in Section 4, the results of the new analytical procedure are shown. In Section 5, a comparison is made between existing analytical procedures, the new analytical procedure and the iterative approach. Finally, conclusions are given in Section 6 .

\section{Iterative solution for the transfer coefficients}

The turbulent fluxes of momentum and sensible heat between the surface and the atmosphere are given by

$$
\begin{aligned}
\left(\overline{u^{\prime} w^{\prime}}\right)_{0}=-u_{*}^{2} & =-C_{\mathrm{M}} u_{a}^{2}, \\
\left(\overline{w^{\prime} \theta^{\prime}}\right)_{0}=-u_{*} \theta_{*} & =-C_{\mathrm{H}} u_{a}\left(\theta-\theta_{s}\right),
\end{aligned}
$$


where $u_{a}$ and $\theta$ are the absolute wind speed and potential temperature at the reference height (or height of the first model level ) $z$ above the displacement height, $\theta_{s}$ is the surface potential temperature, $C_{\mathrm{M}}$ and $C_{\mathrm{H}}$ are the drag coefficients for momentum and sensible heat respectively, $u_{*}$ is the friction velocity, and $\theta_{*}$ is the turbulent temperature scale. Based on MOST (Monin and Obukhov, 1954) and including RSL effects (e.g. Physick and Garratt, 1995; De Ridder, 2010), one assumes surface profiles for wind and temperature. Hereby, the lower boundary conditions, i.e. the zero wind and $\theta=\theta_{s}$, are considered at $z=z_{0}$ and $z=z_{0 \mathrm{H}}$, respectively. The transfer coefficients within this framework can be calculated by:

$$
\begin{gathered}
C_{\mathrm{M}}=k^{2}\left[\ln \frac{z}{z_{0}}-\Psi_{\mathrm{M}}(\zeta)+\Psi_{\mathrm{M}}\left(\frac{z_{0}}{z} \zeta\right)+\Psi_{\mathrm{M}}^{*}\left(\zeta, \frac{z}{z_{*}}\right)\right]^{-2}, \\
C_{\mathrm{H}}=k \sqrt{C_{\mathrm{M}}}\left[\ln \frac{z}{z_{0 \mathrm{H}}}-\Psi_{\mathrm{H}}(\zeta)+\Psi_{\mathrm{H}}\left(\frac{z_{0 \mathrm{H}}}{z} \zeta\right)+\Psi_{\mathrm{H}}^{*}\left(\zeta, \frac{z}{z_{*}}\right)\right]^{-1},
\end{gathered}
$$

where $\zeta=z k g \theta_{*} /\left(u_{*}^{2} \theta_{0}\right)$ is the stability parameter, $\Psi_{\mathrm{M}}$ and $\Psi_{\mathrm{H}}$ are the (integrated) stability functions for momentum and heat, $\Psi_{\mathrm{M}}^{*}$ and $\Psi_{\mathrm{H}}^{*}$ are the profile corrections to account for the RSL, $\theta_{0}$ is a reference potential temperature, $g=9.81 \mathrm{~m} \mathrm{~s}^{-2}$ is the gravitational acceleration, and $z_{*}$ is the roughness sublayer height. These surface-layer transfer coefficients can be calculated once $\zeta$ is known. One can find a relation between $R i_{\mathrm{B}}$ and $\zeta$ (see e.g. Arya, 2001, Section 13.4), that can be solved for $\zeta$ iteratively using e.g. the root finding scheme of Ridders (1979) as described in Press et al. (1992). The ultimate challenge is to find an analytical expression for $\zeta$ that makes the iterative method dispensable.

To conclude this section, the flux-profile relationships used in the iterative solution and for calculating the transfer coefficients are given below. For unstable surface-layer conditions $(\zeta<0)$, the integrated profile functions of Paulson (1970) derived from the profile functions of Dyer (1967) or Businger (1966) are adopted:

$$
\begin{aligned}
& \Psi_{\mathrm{M}}=2 \ln \frac{1+x}{2}+\ln \frac{1+x^{2}}{2}-2 \tan ^{-1} x+\pi / 2, \\
& \Psi_{\mathrm{H}}=2 \ln \frac{1+y}{2},
\end{aligned}
$$

where $x=\left(1-\gamma_{m} \zeta\right)^{1 / 4}$ and $y=\left(1-\gamma_{h} \zeta\right)^{1 / 2}$ and $\gamma_{m}=\gamma_{h}=16$. When including RSL effects (see below), one needs the original non-integrated profile functions as well:

$$
\begin{aligned}
\phi_{\mathrm{M}} & =\left(1-\gamma_{m} \zeta\right)^{-1 / 4} \\
\phi_{\mathrm{H}} & =\left(1-\gamma_{h} \zeta\right)^{-1 / 2}
\end{aligned}
$$


For stable conditions $(\zeta>0)$, integrated profile functions are taken from Cheng and Brutsaert (2005) as recommended by Guo and Zhang (2007):

$$
\begin{aligned}
& \Psi_{\mathrm{M}}(\zeta)=-a \ln \left[\zeta+\left(1+\zeta^{b}\right)^{\frac{1}{b}}\right], \\
& \Psi_{\mathrm{H}}(\zeta)=-c \ln \left[\zeta+\left(1+\zeta^{d}\right)^{\frac{1}{d}}\right],
\end{aligned}
$$

$$
\begin{gathered}
\phi_{\mathrm{M}}=1+a \frac{\zeta+\zeta^{b}\left(1+\zeta^{b}\right)^{\frac{1-b}{b}}}{\zeta+\left(1+\zeta^{b}\right)^{\frac{1}{b}}}, \\
\phi_{\mathrm{H}}=1+c \frac{\zeta+\zeta^{d}\left(1+\zeta^{d}\right)^{\frac{1-d}{d}}}{\zeta+\left(1+\zeta^{d}\right)^{\frac{1}{d}}} .
\end{gathered}
$$

To account for RSL effects, the following approximation for $\Psi_{\mathrm{M}}^{*}$ and $\Psi_{\mathrm{H}}^{*}$ proposed by De Ridder (2010) is adopted:

$$
\Psi_{i}^{*}\left(\zeta, \frac{z}{z_{*}}\right) \simeq \phi_{i}\left[\left(1+\frac{\nu}{\mu z / z *}\right) \zeta\right] \frac{1}{\lambda} \ln \left(1+\frac{\lambda}{\mu \frac{z}{z_{*}}}\right) e^{-\mu \frac{z}{z_{*}}}
$$

where $\lambda \simeq 1.5, \mu=\mu_{M} \simeq 2.59, \mu=\mu_{H} \simeq 0.95$ and $\nu \simeq 0.5$. For both the iterative procedure and the analytical approximation presented below, a ratio of $z_{*} / z_{0}=16.7$ is adopted from Sarkar and De Ridder (2010).

\section{The analytical expression for the transfer coefficients}

The analytical approximation to the transfer coefficients is presented below. In essence, an approximate analytical expression for $\zeta$ is derived as a function of $R i_{\mathrm{B}}, z / z_{0}$ and the ratio $z_{0} / z_{0 \mathrm{H}}$ for unstable and stable conditions, seperately.

3.1 Unstable surface layer

The following analytical expression for the unstable surface layer $\left(R i_{\mathrm{B}}<0\right)$ that accounts for the RSL is proposed:

$$
\zeta=(1+p Q) \frac{\left(L_{0 \mathrm{M}}^{*}\right)^{2}}{L_{0 \mathrm{H}}^{*}} R i_{\mathrm{B}}
$$

where $p=\ln \left(1-R i_{\mathrm{B}}\right)$,

$$
\begin{aligned}
Q= & -0.486+0.219 p-0.0331 p^{2}-4.93 e^{-L_{0 \mathrm{H}}}-3.65 L_{0 \mathrm{H}}^{-1} \\
& +0.38 p L_{0 \mathrm{H}}^{-1}+14.8 L_{0 \mathrm{H}}^{-2}-0.946 p L_{0 \mathrm{H}}^{-2}-10.0 L_{0 \mathrm{H}}^{-3} \\
& +0.392 L_{0 \mathrm{M}} L_{0 \mathrm{H}}^{-1}-0.0840 p L_{0 \mathrm{M}} L_{0 \mathrm{H}}^{-1}+0.368 L_{0 \mathrm{M}} L_{0 \mathrm{H}}^{-2},
\end{aligned}
$$


98 and

$$
\begin{aligned}
& L_{0 i}=\ln \left(z / z_{0 i}\right) \\
& L_{0 i}^{*}=L_{0 i}+\frac{1}{\lambda} \ln \left(1+\frac{\lambda}{\mu_{i} \frac{z}{z_{*}}}\right) e^{-\mu_{i} \frac{z}{z_{*}}} .
\end{aligned}
$$

The coefficients appearing in the expression for $Q$ are found by multiple linear regression similar to Yang et al. (2001). Eq. 14 incorporates the correct limit behaviour for $\zeta \rightarrow 0$, for which the (integrated) flux-profile relationships can be linearized in which the slope at the origin $\left(R i_{\mathrm{B}}, \zeta\right)=(0,0)$ is given by $\left(L_{0 \mathrm{M}}^{*}\right)^{2} / L_{0 \mathrm{H}}^{*}$.

\subsection{Stable surface layer}

For the stable surface-layer $\left(R i_{\mathrm{B}}>0\right)$, the normalized transfer coefficients $C_{\mathrm{M}} / C_{\mathrm{Mn}}$ and $C_{\mathrm{H}} / C_{\mathrm{Hn}}$ have a remarkable behaviour (see Section 4 ). The values change drastically for a change from 'small' to 'large' values of $k B^{-1}$. Therefore, we distinguish between weakly and strongly stable conditions. The weakly stable solution for $\zeta$ is given in Section 3.2.1. Afterwards, it is connected to a strongly stable solution in Section 3.2.2.

\subsubsection{The weakly stable solution}

For weakly stable conditions (small positive $\zeta$ ), we first linearize $\phi_{i}$ and $\Psi_{i}$. Afterwards, the relation between $R i_{\mathrm{B}}$ and $\zeta$ is inverted analytically from which we find an expression for $\zeta$.

As in Yang et al. (2001), a linear approximation for (9)-(10) can be made for weakly stable conditions by putting $\Psi_{i}(\zeta) \simeq-\beta_{i} \zeta$. The relation between $R i_{\mathrm{B}}$ and $\zeta$ now reduces to:

$$
R i_{\mathrm{B}}=\zeta \frac{L_{0 \mathrm{H}}^{*}+S_{0 \mathrm{H}}^{*} \beta_{\mathrm{H}} \zeta}{\left(L_{0 \mathrm{M}}^{*}+S_{0 \mathrm{M}}^{*} \beta_{\mathrm{M}} \zeta\right)^{2}},
$$

where

$$
S_{0 i}^{*}=1-z_{0 i} / z+\left(1+\frac{\nu}{\mu_{i} \frac{z}{z_{*}}}\right) \frac{1}{\lambda} \ln \left(1+\frac{\lambda}{\mu_{i} \frac{z}{z_{*}}}\right) e^{-\mu_{i} \frac{z}{z_{*}}} .
$$

It is now possible to obtain $\zeta$ from Eq. 18 analytically as in Byun (1990) for $z_{0}=z_{0 \mathrm{H}}$ or Yang et al. (2001) for $z_{0} \neq z_{0 \mathrm{H}}$ :

$$
\zeta=\frac{-L_{0 \mathrm{M}}^{*}}{S_{0 \mathrm{M}}^{*} \beta_{\mathrm{M}}}-\frac{B C}{4\left(S_{0 \mathrm{M}}^{*} \beta_{\mathrm{M}}\right)^{3}\left(B^{2}+|C r|\right)}+\frac{B-\sqrt{B^{2}+C r}+\frac{B C r}{2\left(B^{2}+|C r|\right)}}{2\left(S_{0 \mathrm{M}}^{*} \beta_{\mathrm{M}}\right)^{3} r},
$$




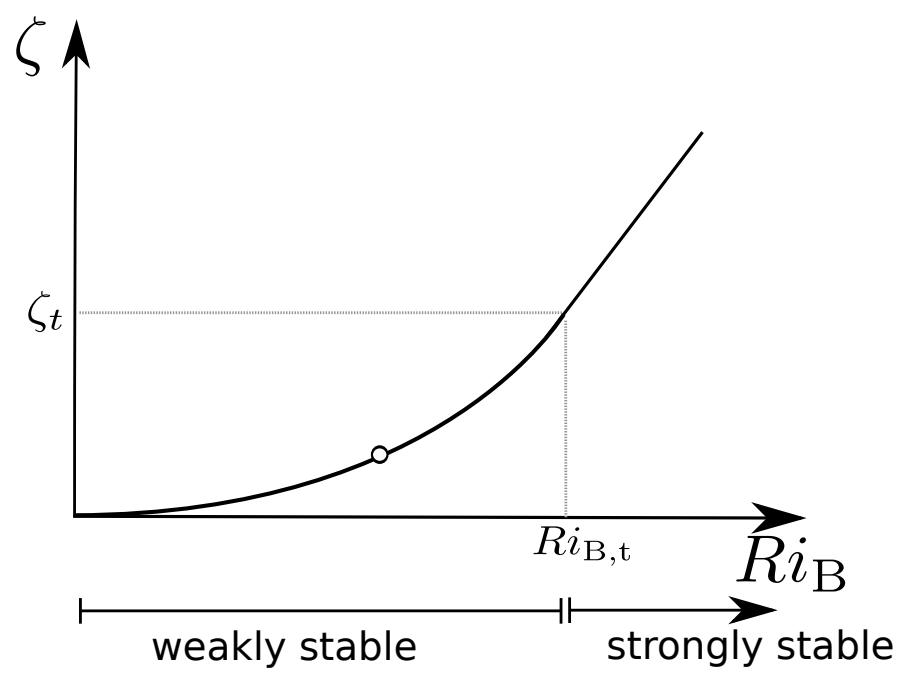

Fig. 1 The analytical approximation of $\zeta$ for the stable surface layer. The intercept of the strongly stable solution $\left(R i_{B, t}, \zeta_{\mathrm{t}}\right)$ that connects the weakly and the strongly stable regimes is shown. The location where $r=0$ in (20) and for which we have to exclude the third term is indicated with an open circle. See text for further information.

where

$$
\begin{aligned}
r & =R i_{\mathrm{B}}-S_{0 \mathrm{H}}^{*} \beta_{\mathrm{H}} /\left(S_{0 \mathrm{M}}^{*} \beta_{\mathrm{M}}\right)^{2}, \\
B & =S_{0 \mathrm{M}}^{*} \beta_{\mathrm{M}} L_{0 \mathrm{H}}^{*}-2 S_{0 \mathrm{H}}^{*} \beta_{\mathrm{H}} L_{0 \mathrm{M}}^{*}, \\
C & =4\left(S_{0 \mathrm{M}}^{*} \beta_{\mathrm{M}}\right)^{2} L_{0 \mathrm{M}}^{*}\left(S_{0 \mathrm{H}}^{*} \beta_{\mathrm{H}} L_{0 \mathrm{M}}^{*}-S_{0 \mathrm{M}}^{*} \beta_{\mathrm{M}} L_{0 \mathrm{H}}^{*}\right) .
\end{aligned}
$$

As suggested by Li et al. (2010), a linear relation between $R i_{\mathrm{B}}$ and $\zeta$ for strongly stable conditions (large positive $\zeta$ ) can be assumed to approximate the iterative solution. Its constant slope $d \zeta / d R i_{\mathrm{B}}$ and its intercept $\left(R i_{B, t}, \zeta_{\mathrm{t}}\right)$, at which the weakly stable solution ceases and the strongly stable solution commences, is now determined. Therefore, it is assumed that the slope of the strongly stable solution $d \zeta / d R i_{\mathrm{B}}$ is equal to the slope of the weakly stable solution at the intercept $R i_{B, t}$. The latter is expressed by:

$$
D\left(\zeta_{t}\right)=\frac{\left(L_{0 \mathrm{M}}^{*}+S_{0 \mathrm{M}}^{*} \beta_{\mathrm{M}} \zeta_{\mathrm{t}}\right)^{3}}{L_{0 \mathrm{M}}^{*} L_{0 \mathrm{H}}^{*}+\zeta_{\mathrm{t}}\left(2 S_{0 \mathrm{H}}^{*} \beta_{\mathrm{H}} L_{0 \mathrm{M}}^{*}-S_{0 \mathrm{M}}^{*} \beta_{\mathrm{M}} L_{0 \mathrm{H}}^{*}\right)},
$$




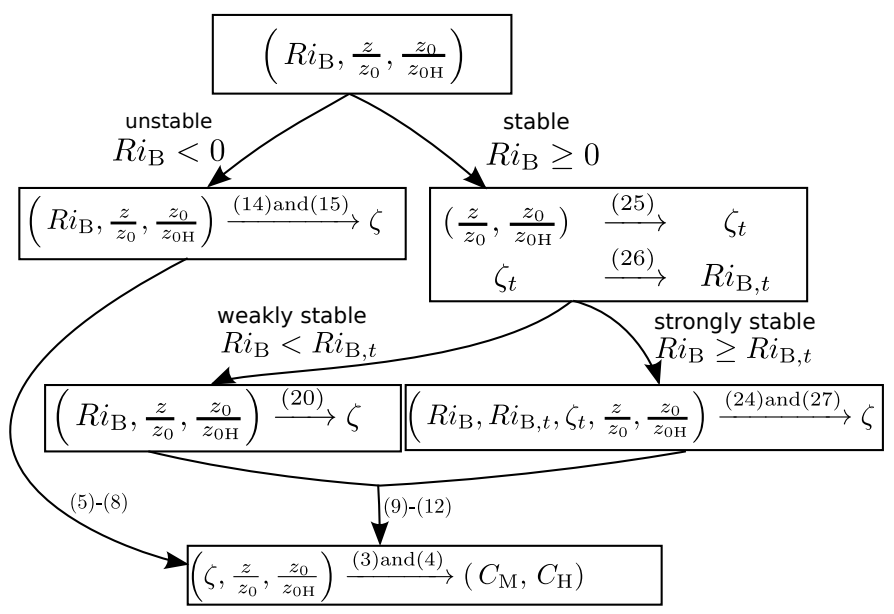

Fig. 2 A decision tree for calculating $\zeta$ and the consequential transfer coefficients

which can be obtained by taking the reciprocal of the derivative of (18). The following two criteria now have to be fulfilled simultaneously for the intercept $\left(R i_{B, t}, \zeta_{\mathrm{t}}\right)$ at which the weakly stable solution ceases and the strongly stable solution commences. Firstly, $\zeta_{\mathrm{t}}$ should be lower than the maximum $\zeta$ for which the weakly stable solution $\left(\zeta<\zeta_{\mathrm{t}}\right)$ is a good approximation. Secondly, $D\left(\zeta_{\mathrm{t}}\right)$ given by (24) should be a representative value for the slope of the strongly stable solution $\left(\zeta \geq \zeta_{\mathrm{t}}\right)$. The following expression for $\zeta_{\mathrm{t}}$ meets these two criteria:

$$
\begin{aligned}
\zeta_{t}= & -0.316-0.515 e^{-L_{0 \mathrm{H}}}+25.8 e^{-2 L_{0 \mathrm{H}}}+4.36 L_{0 \mathrm{H}}^{-1} \\
& -6.39 L_{0 \mathrm{H}}^{-2}+0.834\left(\ln L_{0 \mathrm{M}}\right)-0.0267\left(\ln L_{0 \mathrm{M}}\right)^{2} .
\end{aligned}
$$

Then, $R i_{B, t}$ can be obtained by calculating (18) at $\zeta_{\mathrm{t}}$ :

$$
R i_{B, t}=\zeta_{\mathrm{t}} \frac{L_{0 \mathrm{H}}^{*}+S_{0 \mathrm{H}}^{*} \beta_{\mathrm{H}} \zeta_{\mathrm{t}}}{\left(L_{0 \mathrm{M}}^{*}+S_{0 \mathrm{M}}^{*} \beta_{\mathrm{M}} \zeta_{\mathrm{t}}\right)^{2}} .
$$

The expression for the strongly stable solution $\left(R i_{\mathrm{B}}>R i_{B, t}\right)$ is now given by:

$$
\zeta=\zeta_{\mathrm{t}}+D\left(\zeta_{\mathrm{t}}\right)\left(R i_{\mathrm{B}}-R i_{B, t}\right)
$$

For the weakly stable solution $\left(R i_{\mathrm{B}} \leq R i_{B, t}\right)$, the solution is given by $(20)$. An analytical solution for the whole stable range has now been constructed.

Figure 2 gives an overview of the presented analytical solution for both unstable and stable conditions for easy implementation.

\section{Results}

The normalized transfer coefficients $C_{\mathrm{M}} / C_{\mathrm{M}, \mathrm{n}}$ and $C_{\mathrm{H}} / C_{\mathrm{H}, \mathrm{n}}$, for which ' $\mathrm{n}$ ' refers to neutral stability $\left(R i_{\mathrm{B}}=0\right)$, are compared for the approximate 
Unstable conditions
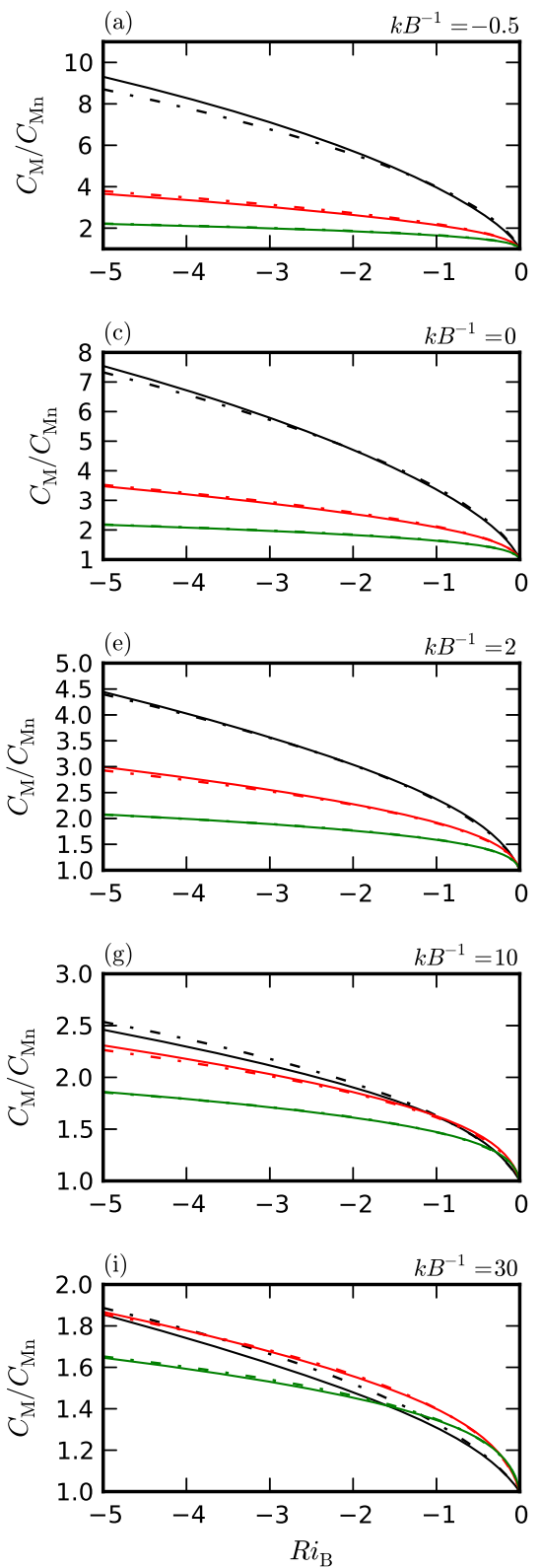

Stable conditions
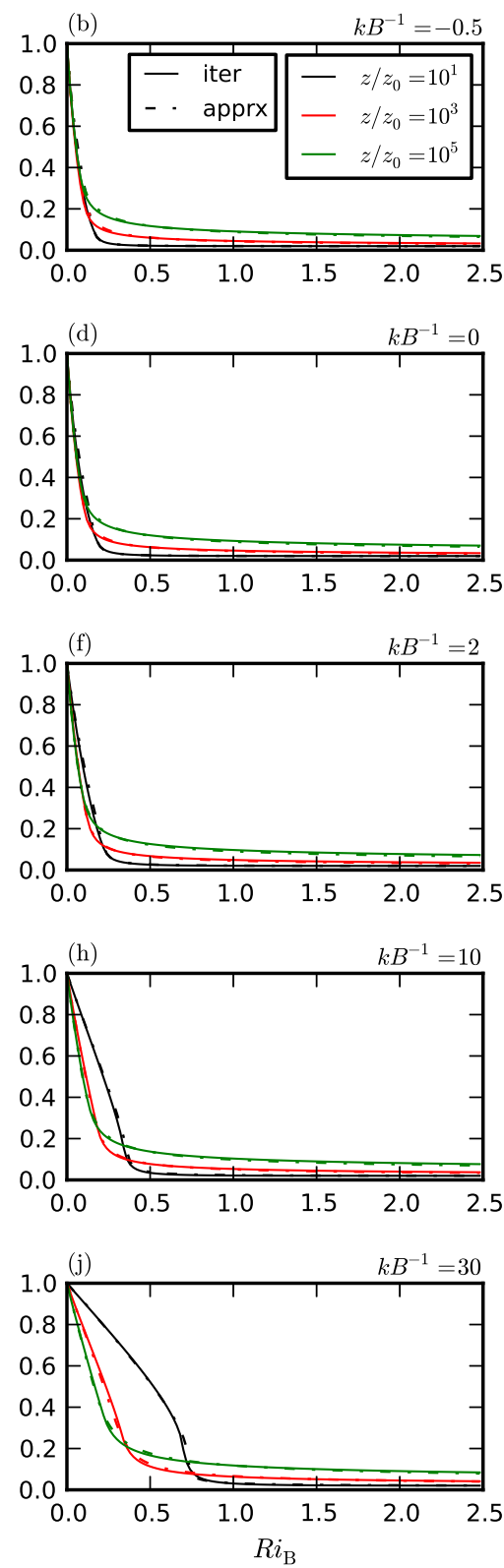

Fig. 3 The approximate (apprx) and iterative (iter) transfer relations for momentum are demonstrated. Unstable and stable conditions are shown respectively on the left in panels (a)-(c)-(e)-(g)-(i)-(k) and on the right in panels (b)-(d)-(f)-(h)-(j)-(l). $k B^{-1}$ is set to -0.5 , $0,2,10,20$ and 30 in panels $(\mathrm{a})-(\mathrm{b}),(\mathrm{c})-(\mathrm{d}),(\mathrm{e})-(\mathrm{f}),(\mathrm{g})-(\mathrm{h}),(\mathrm{i})-(\mathrm{j})$ and $(\mathrm{k})-(\mathrm{l})$, respectively. In each panel, values for $z / z_{0}$ are shown in different colours. 
Unstable conditions
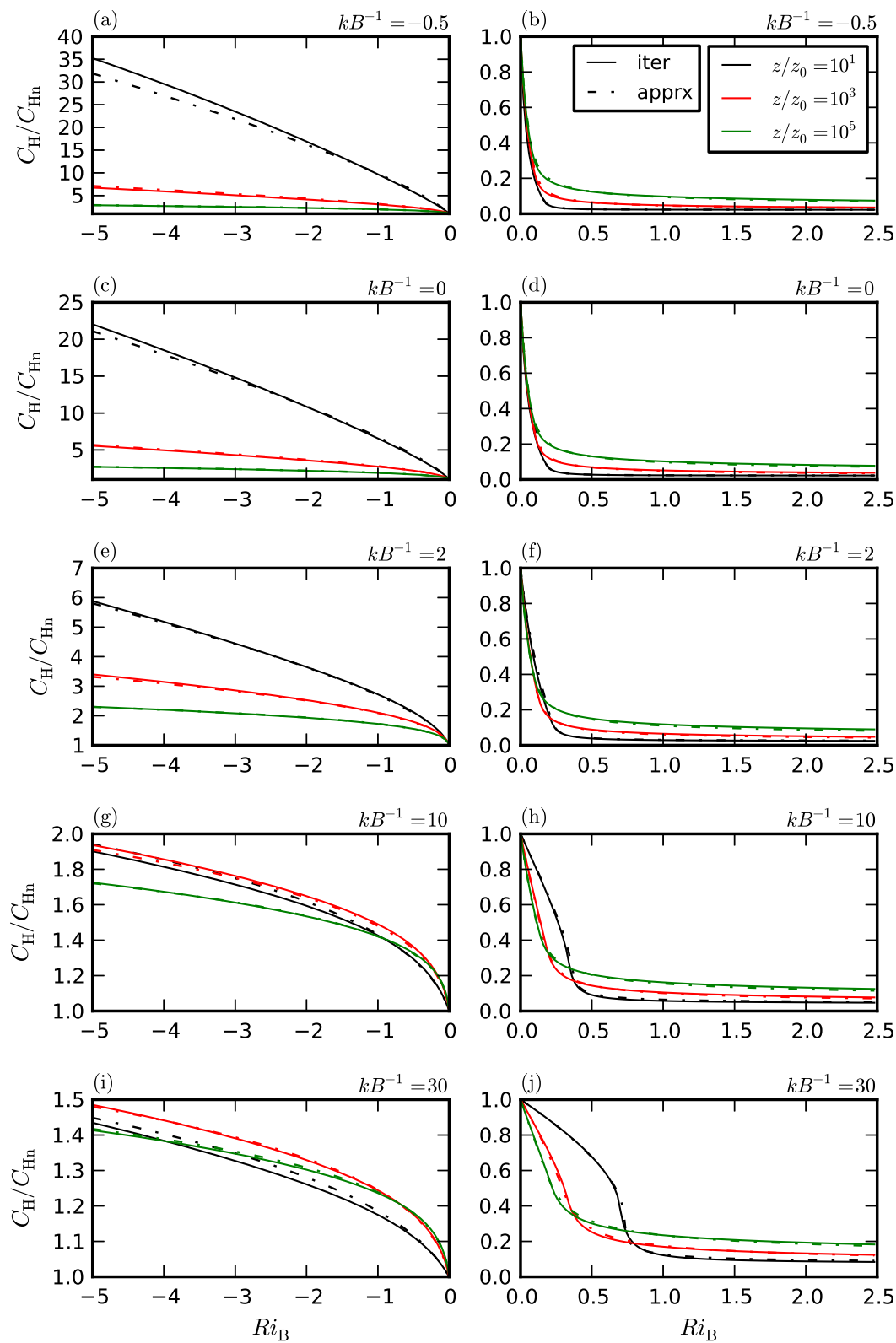

Fig. 4 As in Fig. 3, but for the transfer relations for heat instead of momentum. 
analytical and iterative solution for $k B^{-1}=\{-0.5,0,2,10,20,30\}, z / z_{0}=$ $\left\{10,10^{3}, 10^{5}\right\}$ and $R i_{\mathrm{B}}$ between -5 and 2.5 by steps of 0.02 . The reference height (i.e. height of the first model level) is typically 10 to a few tens of metres, and consequently $z_{0}$ values up to $1.5 \mathrm{~m}$ - typical for urban surfaces - are captured. The resulting transfer relations are shown in Figs 3 and 4, and are discussed below.

For the stable region, the resulting analytical transfer relations follow the iterative solution very well, despite the fact that the functions change shape drastically when going from low to high $k B^{-1}$ values. In particular, they decrease drastically from a certain $R i_{\mathrm{B}}$ value and this value itself depends strongly on the ratio $z / z_{0}$ and $k B^{-1}$. This is captured very well, because the analytical approximation has a variable transition point $R i_{B, t}$ between the weakly stable and the strongly stable regime that depends on $z_{0}$ and $k B^{-1}$. Because of the linear approximation for the strongly stable solution, there can be a substantial deviation between the analytical and iterative solutions for $\zeta$ as a function of $R i_{\mathrm{B}}$ for strongly stable conditions. However, this has a minor effect on the final values of the transfer coefficients, because they are nearly constant for strongly stable conditions. The relative error is less than $10 \%$, except at an $R i_{\mathrm{B}}$-range around $R i_{B, t}$, at which the transfer coefficients fall abruptly with increasing $R i_{\mathrm{B}}$. For this narrow range, the relative error can be up to $40 \%$ for $k B^{-1}>15$. Because of the dramatic drop around $R i_{B, t}$ and the sensitivity of the position for this drop on $z / z_{0}$ and $k B^{-1}$, the error of the analytical approximation around $R i_{B, t}$ is actually small compared to other uncertainties of either $R i_{\mathrm{B}}, z / z_{0}$ or $k B^{-1}$. Moreover, uncertainties in the fluxprofile relationships are much larger than the error of the analytical solution for very stable conditions. This can be seen when comparing the iterative solution obtained with the flux-profile relationships of Beljaars and Holtslag (1991) with that obtained with the flux-profile relationships of Cheng and Brutsaert (2005), see Section 5. For unstable conditions, we obtain good agreement for $C_{\mathrm{M}} / C_{\mathrm{Mn}}$ and $C_{\mathrm{H}} / C_{\mathrm{Hn}}$ : the relative error of the transfer coefficients is always $<10 \%$ for $-5 \leq R i_{\mathrm{B}}<0$.

\section{Discussion: comparison with other non-iterative methods}

A comparison is made between existing non-iterative parametrizations from Yang et al. (2001) and Li et al. (2010), the non-iterative parametrization presented herein and the iterative solution. This is done under conditions for which our parametrization was developed, i.e. urban surfaces. A typical value for the roughness length $\left(z_{0}\right)$ for urban areas is $1 \mathrm{~m}$ and this leads to a ratio $z / z_{0}=15$ when assuming a height of the first model layer of $z=15 \mathrm{~m}$. With this configuration, we discuss the normalized transfer coefficients $C_{\mathrm{H}} / C_{\mathrm{Hn}}$ as a function of $k B^{-1}$ for four stability conditions in Fig. 5 (similar results are found for $C_{\mathrm{M}} / C_{\mathrm{Mn}}$ ).

For the weakly unstable case $\left(R i_{\mathrm{B}}=-0.08\right)$, Li et al. (2010) has a large deviation from the iterative solution for high $k B^{-1}$ values. In contrast, Yang 
(a) $R i_{\mathrm{B}}=-0.08$ and $z / z_{0 \mathrm{M}}=15$

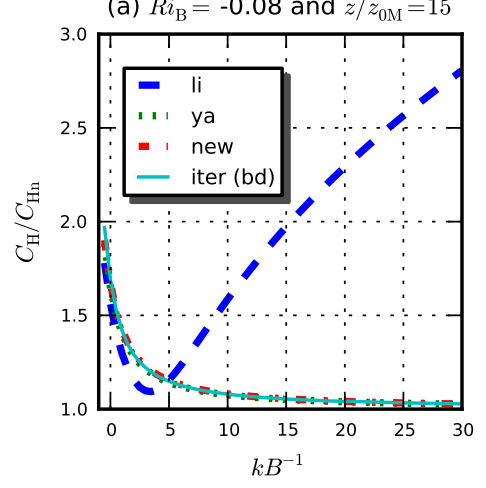

(c) $R i_{\mathrm{B}}=-3.0$ and $z / z_{0 \mathrm{M}}=15$

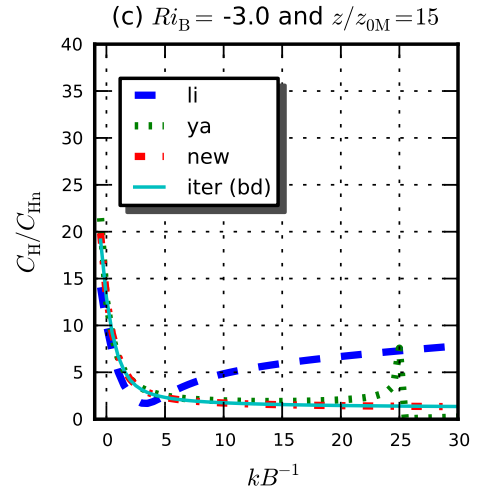

(b) $R i_{\mathrm{B}}=0.08$ and $z / z_{0 \mathrm{M}}=15$

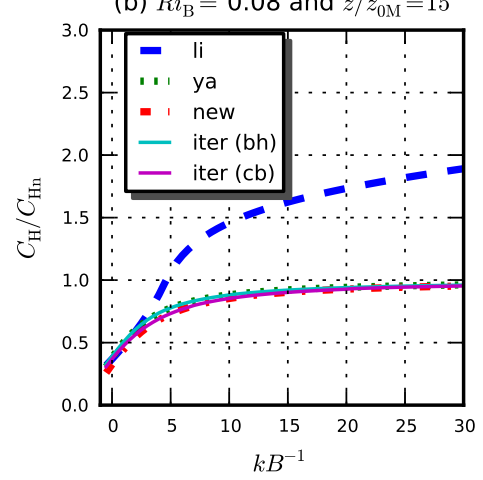

(d) $R i_{\mathrm{B}}=1.0$ and $z / z_{0 \mathrm{M}}=15$

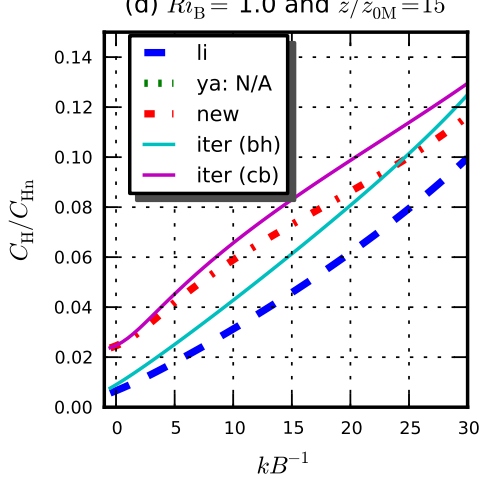

Fig. 5 Normalized transfer coefficients for heat as a function of $k B^{-1}$ between -0.5 and 30 for a fixed value of $z / z_{0}=15$. Weakly unstable $\left(R i_{\mathrm{B}}=-0.08\right)$, weakly stable $\left(R i_{\mathrm{B}}=0.08\right)$, strongly unstable $\left(R i_{\mathrm{B}}=-3\right)$ and strongly stable $\left(R i_{\mathrm{B}}=1\right)$ surfacelayer conditions are demonstrated in panel (a), (b), (c) and (d) respectively. Results for the analytical approximations of Yang et al. (2001) (ya), Li et al. (2010) (li), the new parametrization (new) and the iterative solution (iter) are shown. For unstable stratification, the iterative solution (bd) uses (integrated) flux-profile relationships from Paulson (1970) for the unstable case. For the stable situations, the iterative solutions are shown using fluxprofile relationships from Beljaars and Holtslag (1991) (bh) and from Cheng and Brutsaert (2005) (cb) respectively.

et al. (2001) and the current parametrization compare very well with the iterative solution. For the weakly stable case $\left(R i_{\mathrm{B}}=0.08\right)$, Yang et al. (2001) gives the best comparison with the iterative solution with flux-profile relationships from Beljaars and Holtslag (1991) and the new proposed analytical solution with the flux-profile relationships from Cheng and Brutsaert (2005). A large deviation is found for Li et al. (2010) compared to the iterative solution for $k B^{-1}>4$ : the ratio $C_{\mathrm{H}} / C_{\mathrm{Hn}}$ becomes $>1$, i.e. the drag coefficient for the stable case becomes higher than that for the neutral case which is unrealis- 
tic. For the strongly unstable case $\left(R i_{\mathrm{B}}=-3\right)$, similar results are obtained as in the weakly unstable case. In addition, Yang et al. (2001) has a substantial deviation for very large $k B^{-1}$ values between 20 and 25 , while it is invalid for $k B^{-1}>25$. For the strongly stable case $\left(R i_{\mathrm{B}}=1\right)$, Yang et al. (2001) does not have a solution, but now Li et al. (2010) performs relatively well. Again here, the new parametrization performs well for all $k B^{-1}$ values. As mentioned before, the new analytical approximation for stable stratification is constructed using flux-profile relationships from Cheng and Brutsaert (2005) for stable stratification. In contrast, the analytical approximation of Li et al. (2010) is constructed using those from Beljaars and Holtslag (1991). For the strongly stable case, a substantial difference occurs between the iterative solutions using the different flux-profile relationships, and consequently also in their respective analytical approximations. This results from a large discrepancy between the different flux-profile relationships for strongly stable stratification (Guo and Zhang, 2007). Given this uncertainty for strong stability, the errors made with the new analytical approximation over the iterative solution are negligible.

To conclude, both the analytical approximations of Li et al. (2010) and Yang et al. (2001) perform well for limited ranges of $k B^{-1}$ and $R i_{\mathrm{B}}$ values, but they fail to approximate the iterative solution(s) for the full range $-0.5 \leq$ $k B^{-1} \leq 30$ and $-5 \leq R i_{\mathrm{B}} \leq 2.5$. In particular, large $k B^{-1}$ values typical for urban surfaces are problematic. In contrast, the new non-iterative solution proposed herein captures the full range very well.

\section{Conclusions}

In order to determine turbulent fluxes momentum and sensible heat between the surface and the atmosphere in a mesoscale atmospheric model, a new analytical method is presented to approximate the iterative transfer coefficients within the MOST framework, including RSL effects. Essentially, an analytical expression for $\zeta$ as a function of $R i_{\mathrm{B}}$ is found from which the transfer coefficients for momentum and sensible heat are obtained. The transfer coefficients for other scalar variables such as moisture $(q)$ can be taken equal to the transfer coefficient of sensible heat as shown in e.g. Andreas and Murphy (1986). For the unstable case $\left(R i_{\mathrm{B}}<0\right)$, an $\zeta$ is obtained by multiple linear regression, in which the correct limit behaviour for $R i_{\mathrm{B}} \rightarrow 0$ is incorporated. For weakly stable conditions, the exact solution for $\zeta$ using linear stability functions is used, and for strongly stable conditions a linear relation between $R i_{\mathrm{B}}$ and $\zeta$ is assumed. The weakly and strongly stable solutions are smoothly connected by a transition point $\left(R i_{B, t}, \zeta_{\mathrm{t}}\right)$ that depends on $k B^{-1}$ and the ratio $z / z_{0}$.

The transfer coefficients resulting from this analytical approximation reproduce the iterative solution very well for $10 \leq z / z_{0} \leq 10^{5}$ and $-0.5 \leq$ $k B^{-1} \leq 30$ that represents most, if not all, existing surface types. This should lead to an overall better performance with regard to the momentum exchange and energy balance at the surface. As most existing global and regional at- 
mospheric models use an analytical approach already, our new method will require only a small change to the model code ${ }^{1}$. Especially, the presented bulk parametrization that avoids any iterative procedure can be used to simulate the contrasting behaviour among different land-surface types, for example to study the urban heat island effect as in Van Weverberg et al. (2008), Demuzere et al. (2008) and Sarkar and De Ridder (2010).

Acknowledgements This research has been conducted within a collaboration between VITO and KU Leuven for improving the meteorological forcing for urban-scale air-quality simulations. It was supported by the program Science for a Sustainable Development (SSD) of the Belgian Science Policy Office (BELSPO) under contract number SD/CS/04A. Special thanks go to the anonymous reviewers for their suggestions, in particular for improving the readability of the manuscript. We would also acknowledge Gerd Vogel from DWD for validating initial tests of TERRA-ML standalone with the new transfer coefficients.

\section{References}

Andreas, E. L. and B. Murphy, 1986: Bulk transfer coefficients for heat and momentum over leads and polynas. J Phys Oceanogr, 16, 1875-1883.

Arya, S. P., 2001: Introduction to micrometeorology. 2d ed., Academic Press, San Diego, 420 pp.

Beljaars, A. C. M. and A. A. M. Holtslag, 1991: Flux Parameterization over Land Surfaces for Atmospheric Models. J Appl Meteorol, 30, 327-341.

Blümel, K., 2000: An Approximate Analytical Solution of Flux-profile Relationships for the Atmospheric Surface Layer with Different Momentum and Heat Roughness Lengths. Boundary-Layer Meteorol, 97, 251-271.

Businger, J. A., 1966: Transfer of momentum and heat in the planetary boundary layer. Proc. Symp. Arctic Heat Budget and Atmospheric Ciculation, 305331.

Byun, D. W., 1990: On the Analytical Solution of Flux-Profile Relationships for the Atmospheric Surface Layer. J Appl Meteorol, 29, 652-657.

Cheng, Y. G. and W. Brutsaert, 2005: Flux-profile relationships for wind speed and temperature in the stable atmospheric boundary layer. Boundary-Layer Meteorol, 114, 519-538.

De Bruin, H. A. R., R. J. Ronda, and B. J. H. Van De Wiel, 2000: Approximate Solutions For The Obukhov Length And The Surface Fluxes In Terms Of Bulk Richardson Numbers. Boundary-Layer Meteorol, 95, 145-157.

De Ridder, K., 2010: Bulk Transfer Relations for the Roughness Sublayer. Boundary-Layer Meteorol, 134, 257-267.

Demuzere, M., K. De Ridder, and N. P. M. van Lipzig, 2008: Modeling the energy balance in Marseille: Sensitivity to roughness length parametrizations and thermal admittance. J Geophys Res, 113, 1-19.

Dyer, A. J., 1967: The turbulent transport of heat and water vapour in an unstable atmosphere. $Q J R$ Meteorol Soc, 93, 501-508.

1 The Fortran code for the presented non-iterative approximation can be obtained from the authors on request. 
Garratt, J. R., 1992: The atmospheric boundary layer. Cambridge University Press, UK, 316 pp.

Guo, X. and H. Zhang, 2007: A performance comparison between nonlinear similarity functions in bulk parameterization for very stable conditions. Environmental Fluid Mechanics, 7, 239-257.

Holtslag, A. A. M. and M. Ek, 1996: Simulation of Surface Fluxes and Boundary-Layer Development over the Pine Forest in HAPEX-MOBILHY. J Appl Meteorol, 35, 202-213.

Launiainen, J., 1995: Derivation of the relationship between the Obukhov stability parameter and the bulk Richardson number for flux-profile studies. Boundary-Layer Meteorol, 76, 165-179.

Lee, H. N., 1997: Improvement of Surface Flux Calculations in the Atmospheric Surface Layer. J Appl Meteorol, 36, 1416-1423.

Li, Y., Z. Gao, D. H. Lenschow, and Z. F. Chen, 2010: An Improved Approach for Parameterizing Surface-Layer Turbulent Transfer Coefficients in Numerical Models. Boundary-Layer Meteorol, 137, 153-165.

Louis, J.-F., 1979: A Parametric Model of the Vertical Eddy Fluxes in the Atmosphere. Boundary-Layer Meteorol, 17, 187-202.

Mascart, P., J. Noilhan, and H. Giordani, 1995: A modified parameterization of flux-profile relationships in the surface layer using different roughness length values for heat and momentum. Boundary-Layer Meteorol, 72, 331-344.

Monin, A. S. and A. M. Obukhov, 1954: Dimensionless Characteristics of Turbulence in the Surface Layer of the Atmosphere. Trudy Geofiz Inst Akad Nauk SSSR, 24, 163-187.

Paulson, C. A., 1970: The Mathematical Representation of Wind Speed and Temperature Profiles in the Unstable Atmospheric Surface Layer. $J$ Appl Meteorol, 9, 857-861.

Physick, W. L. and J. R. Garratt, 1995: Incorporation of a high-roughness lower boundary into a mesoscale model for studies of dry deposition over complex terrain. Boundary-Layer Meteorol, 74, 55-71.

Pleim, J. E., 2006: A Simple, Efficient Solution of Flux-Profile Relationships in the Atmospheric Surface Layer. J Appl Meteor Climatol, 45, 341-347.

Press, W. H., S. A. Teukolsky, W. T. Vetterling, and B. P. Flannery, 1992: Numerical recipes in FORTRAN: The art of scientific computing. $2 \mathrm{~d}$ ed., Cambridge University Press, 934 pp.

Ridders, C. J. F., 1979: A new algorithm for computing a single root of a real continuous function. IEEE Trans Circuits Syst, 26, 979-980.

Sarkar, A. and K. De Ridder, 2010: The Urban Heat Island Intensity of Paris: A Case Study Based on a Simple Urban Surface Parametrization. BoundaryLayer Meteorol, 138, 511-520.

Song, Y., 1998: An Improvement of the Louis Scheme for the Surface Layer in an Atmospheric Modelling System. Boundary-Layer Meteorol, 88, 239-254.

Sugawara, H. and K. Narita, 2008: Roughness Length for Heat over an Urban Canopy. Theor Appl Climatol, 95, 291-299.

Uno, I., X. M. Cai, D. G. Steyn, and S. Emori, 1995: A simple extension of the Louis method for rough surface layer modeling. Boundary-Layer Meteorol, 
76, 395-409.

312 van den Hurk, B. and A. Holtslag, 1997: On the bulk parameterization of surface fluxes for various conditions and parameter ranges. Boundary-Layer Meteorol, 82 (1), 119-133.

Van Weverberg, K., K. De Ridder, and A. Van Rompaey, 2008: Modeling the Contribution of the Brussels Heat Island to a Long Temperature Time Series. J Appl Meteor Climatol, 47, 976-990.

Viterbo, P., A. C. M. Beljaars, J.-F. Mahouf, and J. Teixeira, 1999: The representation of soil moisture freezing and its impact on the stable boundary layer. Q J R Meteorol Soc, 125, 2401-2426.

Voogt, J. A. and C. S. B. Grimmond, 2000: Modelling surface sensible heat flux using surface radiative temperatures in a simple urban area. $J \mathrm{Appl}$ Meteorol, 39, 1679-1699.

Yang, K., N. Tamai, and T. Koike, 2001: Analytical Solution of Surface Layer Similarity Equations. J Appl Meteorol, 40, 1647-1653. 